\title{
A METHOD FOR ISOLATING THE RENAL TUBULES OF MAMMALIA
}

\author{
G. CARL HUBER
}

University of Michigan

ONE PLATE

Two methods are available for ascertaining the size, shape, sequence and relations of the several parts of a renal tubule of mammalia, namely, reconstruction by the wax-plate method and maceration of kidney substance to an extent permitting isolation of tubules or tubule segments. Of these two methods that of reconstruction is the more satisfactory, within the limits of its applicability, since it permits of the reproduction of the several parts of a tubule in their normal relation and in permanent form. However, the complexity of the renal tubules, especially of adult mammals is such, that it is necessary to have for purposes of wax-plate reconstruction, first, an unbroken and relatively long series of sections, extending through cortex and medulla, and not to exceed 5 micra in thickness, and second, to use a magnification of about 400 diameters. The limits of applicability of the wax-plate method, therefore, is reached about with renal tubules taken from the kidneys of newly born or relatively young stages of the smaller mammals; the renal tubules of newly born rabbits and cats, thus reconstructed resulting in models reaching a length of between four and five feet. The size of the model which would result from reconstruction of a renal tubule of an adult mammal, but more especially the difficulty of tracing through an unbroken series of 150 to 250 sections the different parts of such a tubule, would relegate this method, under such conditions, to the position of one of the most difficult of technical procedures. The method of maceration is the older and has been very widely used. The rapidity and ease of its application, and the fact that large numbers of tubules or tubule segments are readily placed at the disposal of the observer, commend it. However, the methods of maceration as now very generally used are not without limitations, so that while the results of nearly all investigations dealing with form and relations of the several parts of the mammalian renal tubule are based on observations made with the aid of maceration methods, it has not been possible until very recently to even approach the isolation of a complete renal tubule in an adult mammal, all conceptions of their form and relations being 
based on observations on shorter or longer tubule segments, a fact which readily accounts for the diversity of opinions as to many details in regard to form and structure current in literature. As macerating media, hydrochloric and nitric acid have been extensively used, especially the former. With arsenious acid, as recommended by Golgi, I have had only in different results. It is not my purpose, nor is it necessary to review the various methods and macerating media which have been used for the purpose of isolating the renal tubules; further than to call attention to the methods used by Peter ${ }^{1}$ and Zarnik, ${ }^{2}$ whose results deserve special consideration. Peter in a recent and most excellent contribution on the structure of the mammalian kidney, in which he gives an account of extended observations made largely on macerated material, made use of the hydrochloric acid method in the following way: Fresh and relatively large pieces of kidney substances were placed in concentrated hydrochloric acid solution. Hydrocloric acid of sp. gr. 1.124, that is, $24 \% \mathrm{H} \mathrm{Cl}$ was used either concentrated or 75 parts of the acid with 25 parts of distilled water. In this the pieces remained for from several hours to two days, the time varying with the size of the piece and other factors. When the desired degree of maceration was reached the pieces were rinsed several times in distilled water and then kept in the same. Pieces are now and then ready for teasing as soon as placed in the distilled water or not until after a stay in the water varying for from several to 48 hours. By means of this method it would seem that Peter obtained very complete maceration admitting of the isolation of relatively long tubule segments, as is attested by his excellent figures, among them a figure of a short, though practically complete renal tubule from the kidney of an adult mouse (fig. 1, plate 1, of his article) $5 \mathrm{~mm}$. in length; which was, however, so unfortunately mounted that while it admits of the determination of the sequence of the several parts of a renal tubule there is presented no idea of their relation. Peter's diagrams and his statements concerning the length of the renal tubules of man and the other mammals investigated, excellent and accurate as they are, must be regarded as deductions drawn from an observation of relatively long tubular segments, and are not based on actual observation of complete renal tubules. Zarnik investigated the kidney structure of echidna and reptilia. The method used by him is essentially that of Peter, slightly modified as follows: Pieces of kidney, or the entire kidney if small, were placed in concentrated hydrochloric acid (strength not given) for one hour; transferred to distilled water for one hour; again placed in the acid for one hour and then placed in distilled water and kept in the same. To prevent the formation of fungus, the pieces were placed, after a stay of 12 hours in the water, in a solution consisting of 1 per cent formalin and 2 per cent chloral hydrate in dis-

\footnotetext{
${ }^{1}$ Peter, K-Untersuchungen über Bau und Entwickelung der Niere. Fischer, Jena, 1909.

'Zarnik, B-Vergleichende Studien über den Bau der Niere von Echidna und der Reptilienniere. Jenaisch. Zeitsch. f. Naturwissenschaft, Bd. 46, 1910.
} 
tilled water, in which solution they may be kept for months. I shall refer here only to that portion of his contribution dealing with the structures of the kidney of echidna aculeata. The somewhat accidental selection of this form must be regarded as fortunate since the kidney of echidna appears peculiarly favorable for purpose of maceration, presenting as it does, stuctural peculiarities characteristic of the foetal kidney of the higher mammals, so that after maceration for a comparatively short time Zarnik was able to isolate many entire renal tubules although he regrets his inability to mount them in permanent form. The tubules described and figures present in the main the parts described for a mammalian renal tubule. No exact measurements are given for the complete tubules figured. The lengths given, admittedly not exact for the longest tubules, vary from $10 \mathrm{~mm}$. to $30 \mathrm{~mm}$., including if I interpret him correctly, the collecting ducts. The short renal tubules of the mouse figured by Peter and the several examples of the relatively simple renal tubules of echidna figured by Zarnik, constitute, so far as I have been able to ascertain the most successful attempts at the isolation of the complete renal tubules of adult mammalia. I should question the possibility of a more thorough maceration of kidney substance and a more complete isolation of renal tubules by the methods of Peter and Zarnik than was obtained at their hands.

It seemed desirable, therefore, to attempt an extension of the methods of maceration in their application to kidney tissue, more particularly to devise some means of obtaining a more uniform maceration throughout the whole mass, a method by means of which cortex and medulla might be more equally macerated as regards to time. The question of isolating complete renal tubules of adult mammals, appeared to me to depend on the possibility of obtaining complete and uniform maceration of the kidney substance without softening the tubules to an extent making manipulation impossible. This has in a great measure been obtained by injecting concentrated hydrochloric acid solutions into the blood vessels under moderately high pressure. It is the purpose of this note to describe the method devised, the results attainable being portrayed by means of several figures which represent camera lucida drawings of types of tubules isolated and permanently mounted. The method has been tested on the renal tissue of rabbits, cats, dogs, guinea pigs, and rats. From all of which it has been possible to isolate complete renal tubules if one may regard a tubule extending from the renal corpuscle to its junction with the collecting duct as an entire tubule, namely, that portion of a renal tubule which develops from a renal vesicle. Both Peter and Zarnik in their descriptions and in the measurements given have regarded the collecting tubules or ducts as constituting a part of the renal tubules. It would seem, however, more desirable to follow development and distinguish between the renal tubules proper and the collecting ducts. The method, for want of material has not been sufficiently tested on human renal tissue to enable me to consider at the present time the possibility of isolating entire human renal tubules. This is under contemplation. 
Method: For purpose of maceration, I have used almost wholly solutions of hydrochloric acid made by mixing 75 parts of $\mathrm{H} \mathrm{Cl}$, sp. gr. 1.21, with 25 parts of distilled water. This makes a solution somewhat stronger than that used by Peter. The few trials with nitric acid have not been satisfactory. Only fresh tissue has been used. After anaesthesia, not carried to cessation of heart beat, the large vessels of the neck are cut and the animal suspended head downward to admit of free bleeding. The abdomen is then opened and a large cannula inserted in the aota, slightly above the origin of the renal arteries and the cannula filled with normal salt solution or distilled water. A pressure of from $10 \mathrm{lbs}$. to $15 \mathrm{lbs}$. as registered by the pressure gauge, was employed to inject the acid solution by means of an apparatus previously described (G. Carl Huber, The Arteriolae Rectae of the Mammalian Kidney, Amer. Jour. Anat., vol. 6, '06-'07, page 393). It is important to cause the acid to flow into the tissue as rapidly as possible and under relatively high pressure. Successful injections of the cortex are readily made. It is, however, quite difficult to obtain complete injection of the medulla, more particularly of the renal pyramid. The extent of the injection can usually be readily seen on a cut surface, the injected portions presenting a bleached appearance, the uninjected a more normal pink color. The kidney is allowed to remain in place for from 10 minutes to 15 minutes after the injection is completed, when it is removed, denuded of its capsule and cut in several pieces, the number depending on the size of the organ. In the rabbit and the cat it has been usual to cut the kidney twice crosswise, thus obtaining a central segment, including the renal pyramid and having a thickness of about $1 \mathrm{~cm}$. at the cortical border. Obviously, this portion includes the straightest and longest tubules. The two end pieces are cut through the middle in the sagittal plane of the kidney. The pieces thus obtained are placed in a hydrochloric acid solution of the same strength as that used for the injection, using a relatively large quantity, about $150 \mathrm{~cm}$. for one kidney of a rabbit. The pieces, which sink gradually, remain in the solution for from three to four hours. An exact statement concerning the time required cannot be made, the maceration being no doubt influenced by factors not wholly under control. So far as I have been able to determine, successful macerations are more dependent on a thorough injection of the hydrochloric acid than on the length of time which this solution is allowed to act after the injected tissue is placed in the same. The removal of the pieces from the acid is best accomplished by carefully pouring off the greater part of the acid, leaving only a sufficient quantity to allow the pieces to float. The dish is then filled with distilled water. This procedure is followed several times, or until practically all of the acid has been removed. After a stay in the distilled water of from 15 minutes to 20 minutes, during which time the pieces lose their transparency and become quite firm, they are transferred by means of a section lifter to fresh distilled water in which they remain several hours, a longer stay is, however, not injurious. From the distilled water the pieces are transferred to an alum hematoxylin solution-Mayer's hemalaun-but, before doing so it has 
been found advantageous to tease carefully the larger pieces, so as to obtain small pyramidal segments of renal tissues, extending through all of the layers and having a diameter of about $3 \mathrm{~mm}$. at the cortical border. These smaller pieces remain in the hematoxylin solution for from 24 hours to 48 hours; they, however, may remain in this stain for weeks without injury. Staining the smaller pieces, before final teasing, as here suggested is to be recommended and for several reasons: The staining solution appears to neutralize the acid remaining in the tissue; the course of the tubules is much more easily followed in stained than in unstained tissue during teasing and since the delicacy of the isolated tubule is such that their staining after isolation is practically excluded, the desirability of having stained preparations when finally mounted in glycerine is evident. Stained tubules are readily followed under low magnification, and admit of study under higher powers, by use of which the size and arrangement of the nuclei and to some extent their structures may be acertained. For a final teasing thoroughly cleaned Syracuse watch-crystals are used. These are nearly filled with distilled water to which a trace of ammonium hydrate is added-10 to 15 drops of a 0.5 per cent solution of ammonium hydrate in distilled water to each watch-crystal. The ammonia present develops the stain and softens slightly the tissue, so that the tubules become quite pliable. The hematoxylin solution causes the tissues to become quite brittle, this the trace of ammonia rectifies without effecting the tensile strength of the tubules. The teasing is carried on under the stereoscopic binocular and with very fine needles. Under favorable conditions one may readily isolate relatively long tubule segments and now and then an entire tubule. In the course of this study somewhat over 100 tubules extending from the renal corpuscle to the collecting duct have been isolated. It is, however, extremely difficult to mount such tubules in permanent form. Various methods have been tried without obtaining uniformly satisfactory results. With the following procedure it has been impossible to mount certain of the tubules isolated. A tubule which has been completely isolated, and preferably one in which the distal convoluted portion is not completely separated from the renal corpuscle or the coil complex of the proximal convoluted portion, is slowly drawn into a pipette with opening nearly as large as the lumen, the operation being controlled under the binocular, and is transferred to a small dish containing distilled water, and placed under the binocular. A thoroughly cleaned coverglass, $25 \mathrm{~mm}$., by $20 \mathrm{~mm}$. held at one end with coverglass forceps is inserted at an angle until almost completely submerged. The tubule is then worked onto the cover glass to the region where surface of water and cover glass meet. Then on slowly withdrawing the cover glass, it is now and then possible to cause the coil complex of the tubule to adhere to it. If this is accomplished, the further withdrawal of the cover, which needs to be done very gradually and evenly, will cause the medullary loop to arrange itself fairly regularly on the cover glass. After successful transfer of the tubule to the cover glass, this is placed for a few moments at an angle of about $45^{\circ}$ to allow the adherent fluid to drain, the larger drops which collect at the 
bottom are then removed with filter paper. Before the mounting can be completed it is necessary to allow of sufficient evaporation to cause the tubule to adhere to the cover glass, when it may be lowered, begining with the edge nearest the coil complex, on a large drop of glycerine 'drawn out to the length of the cover glass. The glycerine is allowed to spread out very slowly. The preparation may then be sealed with asphalt. Of the tubules isolated only a few have been successfully mounted.

On the accompanying plate there are presented three types of renal tubules isolated from the kidneys of adult rabbits. The tubules here figured are permanently mounted in glycerine. The figures shown represent camera lucida drawings made at a magnification of 100 diameters, reduced five times in the reproduction. Tubule $A$, (fig. 1) which was isolated with a portion of the collecting duct, represents a tubule the renal corpuscle and coil complex of which were situated at the extreme periphery of the cortex. In tubule $B$, (fig. 2) the renal corpuscle and proximal coil complex were situated in the deeper portion of the outer half of the cortex. In tubule $C$, (fig. 3) the renal corpuscle and proximal coil complex were situated in the deepest portion of the cortex just above the medulla. Tubules $A$ and $B$, I was able to transfer to the cover glass and mount in the form in which they were isolated by teasing. Tubule $C$, was successfully transferred to the cover glass after which it was found that a short segment of the distal, the thicker arm of the medullary loop adhered to the surface of the layer of water which had collected on the cover. In an endeavor to depress this portion of the tubule by means of a needle, the tubular segment in question adhered to the needle and was accidently separated. The region is indicated by the dotted lines. The tubules figured are not from the same kidney nor even from the same animal. The figures are placed on the plate with reference to the medullary zones and stripes described by Peter. This author has shown, and I cannot but regard this as a very distinct contribution on his part, that the substantia medullaris may be divided into an inner and outer zone and the outer zone further into an inner and outer stripe, each zone and stripe containing definite segments of the renal tubules; zones and stripes may often be observed in fresh material and even in fixed tissues. The boundary line between the inner and outer, zones occurs in the region of the transition of the thin and transparent epithelium of the medullary loops to that of the dark thicker epithelium of the distal arms of the loops. The boundary between the inner and outer stripes of the outer zone is to be found in the region of transition of the thick medullary segments of the proximal arms to the thin segments of the medullary loops. The dotted line a-a, indicates approximately the boundary line between inner and outer stripes of the outer zone, with reference to which the tubules were given their relative position. The dotted line $b-b$, indicates approximately the boundary line between inner and outer zones. In obtaining the measurements here given I have made use of the method employed by me in ascertaining the length of renal tubules isolated by reconstruc- 
tion, namely, of measuring the length of the tubule as found in the model and dividing the resultant figure by the magnification. This method is perhaps somewhat less accurate when applied to a figure, since in a figure it is difficult to represent exactly the length of loops which pass upward or downward in the line of vision. The measurements given are, therefore, rather too short, since such loops are as a rule somewhat fore-shortened. Peter, who has used the same method in ascertaining the length of the tubular segments isolated by him, has also called attention to this source of error. In the accompanying table of measurements taken, attention was given to the relative lengths of the tubular segments showing characteristic and specifically differentiated epithelium. The expression "to the level of the renal corpuscle" found in several of the column headings, has reference to the figures, and not to the actual relations maintained by the different parts of the respective tubules before teasing. In the first column "proximal convoluted portion from renal corpuscle to level of renal corpuscle" is to be interpreted as representing the length of the proximal convoluted portion of the respective tubules, begining in each with renal corpuscle following it through its several windings until the tubule returns to about the level of the renal corpuscle as seen in the several figures. The measurements given are approximate only to the extent of the very slight variation of the position of the several parts of each tubule as given in the figures from that which they obtained in the tubules before teasing and mounting. The lengths of the several parts of the renal tubules figured are as follows:

\begin{tabular}{|c|c|c|c|c|c|c|c|}
\hline & 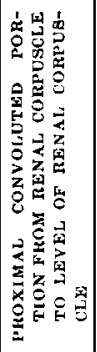 & 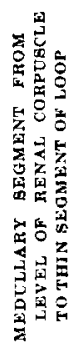 & 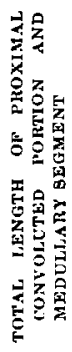 & 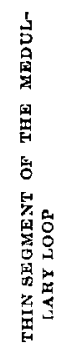 & 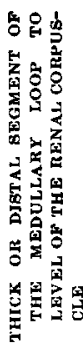 & 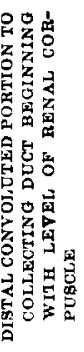 & 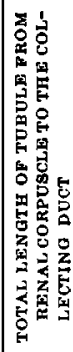 \\
\hline & $\mathrm{mm}$ & $\mathrm{mm}$. & $m m$ & $m m$. & $m m$. & $m m$ & $m m$. \\
\hline $\begin{array}{l}\text { Tubule A, fig. } 1 \\
\text { Tubule B, fig. } 2\end{array}$ & $\begin{array}{l}6.5 \\
5.6\end{array}$ & $\begin{array}{l}4.5 \\
3.8\end{array}$ & $\begin{array}{r}11.3 \\
9.4\end{array}$ & & $\begin{array}{l}6.0 \\
6.0\end{array}$ & $\begin{array}{l}1.8 \\
0.9^{*}\end{array}$ & 23.0 \\
\hline Tubule C, fig. 3 & 7.7 & 2.5 & 10.2 & 15.0 & 2.6 & $1.0^{*}$ & 28.8 \\
\hline
\end{tabular}

*Not traced quite to the collecting duct as may be seen from figures.

It is the primary purpose of this note to call attention to a method for isolating the complete renal tubules of adult mammals and to merely indicate by way of several figures of type-tubules the results which 
may be obtained. A fuller discussion of the mammalian renal tubule is, therefore, withheld for the present. It may be stated, however, that tubules in which the renal corpuscle and proximal coil complex occupy essentially the same relative position in the cortex as those here figured present essentially the same general characteristics as the ones selected for consideration. Those selected represent type-tubules, $A$ and $C$, extremes with $B$, holding an intermediate position. A larger series of tubules selected with reference to position of the renal corpuscle and proximal coil complex of the respective tubules at various levels of the cortex would give a series of figures which would show a gradual transition from a tubule of the type of tubule $A$, to one of the type of tubule $C$. These statements pertain not only to the tubules of the kidney of the rabbit but are equally pertinent to the renal tubules of other mammalian forms studied, with the understanding that the special characteristics of form and structure of renal tubule of the mammal under discussion be considered.

Attention may be drawn to the difference in the relative length of the tubule segments lined with epithelia presenting characteristics, structural peculiarities. I have previously drawn attention to the fact that at least four types of epithelium are to be found in the renal tubules of mammals. These are: $(a)$ the pavement glomerular epithelium; (b) the characteristic renal epithelium of the proximal convoluted portion with its medullary segment; $(c)$ the clear, pavement epithelium of the medullary loop; $(d)$ the thicker, darker epithelium of the distal, the ascending arm of the medullary loop and the distal convoluted portion to near the collecting duct, the later regions presenting perhaps more than one type of epithelium. The measurements above given go to show that the length of those portions of the renal tubules lined by the specific renal epithelium of the cortex, namely, the proximal convoluted portions with their medullary segments, represent tubular segments which do not vary appreciably in length in the tubules of the various types, that, however, the thin tubular segments lined by the clear transparent epithelium of the pavement type vary in length within wide limits in renal tubules of different types. If one may assume a specific excretion for the special renal epithelium lining the proximal convoluted portions and their medullary segments, it is evident that this specific excretion would be essentially of the same extent for all of the renal tubules, irrespective of type. If on the other hand one may assume an absorbtion of water and perhaps certain salts in the segments lined by the clear, pavement epithelium the extent of this absorption must differ widely in tubules presenting short, intermediate and long tubular segments having this structure, and that, therefore, urine of different degrees of concentration must as a consequence enter the collecting tubules.

An outline of the method as here given was presented at the 1910 , October, meeting of the Research Club of the University of Michigan, with a demonstration of certain of the tubules figured. A fuller account, accompanied by demonstrations, was presented at the Ithaca meeting, December, 1910, of the American Association of Anatomists. 


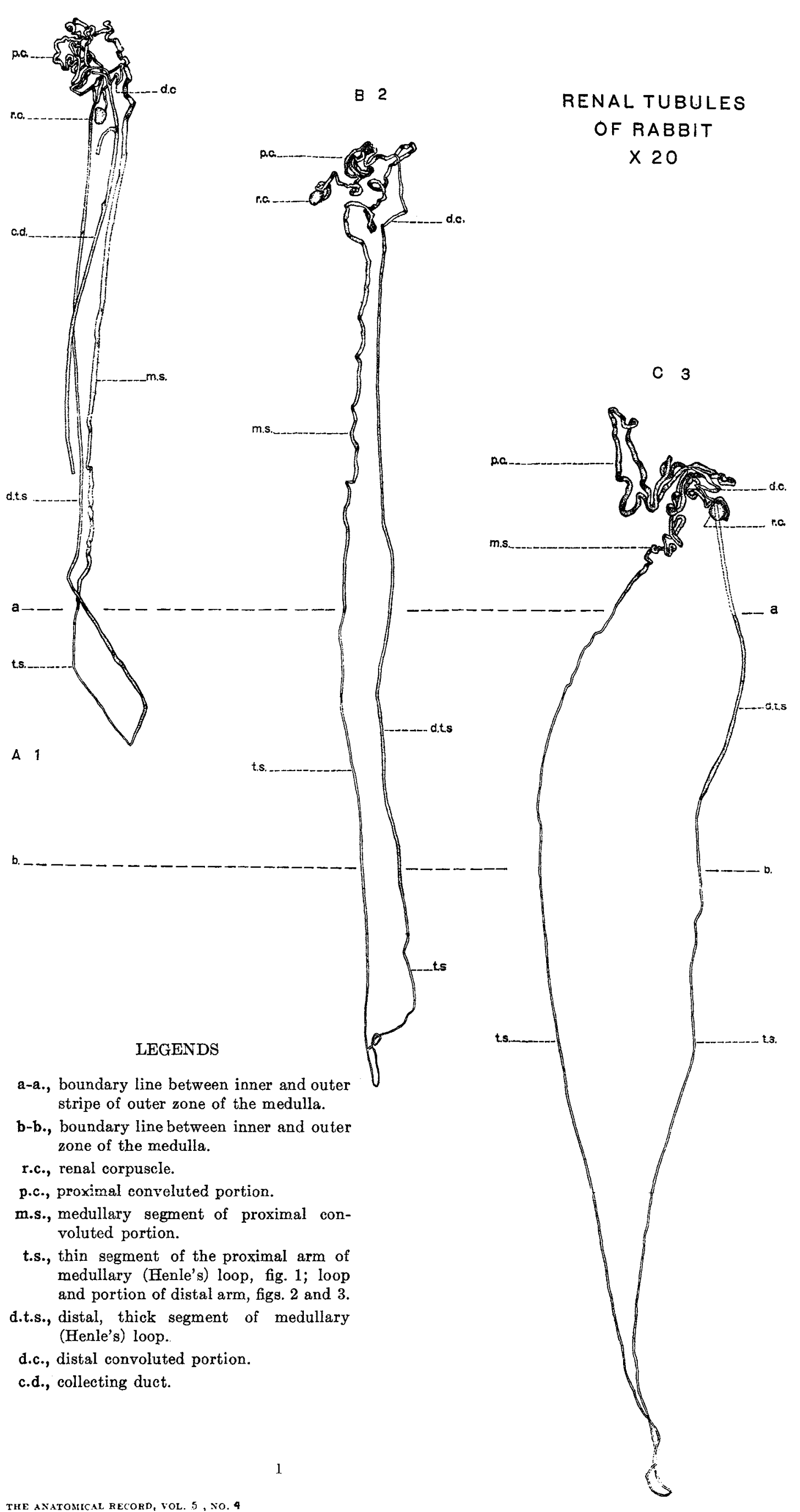

